\title{
CONSTRUÇÃO CIVIL E A CERTIFICAÇÃO AMBIENTAL: ESTUDO DA IMPORTÂNCIA DA SUSTENTABILIDADE EM CONSTRUÇÕES CIVÍS BASEADO NA CERTIFICAÇÃO LEED
}

Felipe Ferreira Cossich ${ }^{1}$, Berna Valentina Bruit ${ }^{2}$, Cleiltan Novais da Silva ${ }^{1}$, Luciana Cristina Soto Herek Rezende ${ }^{1,3}$, Jose Eduardo Gonçalves ${ }^{1,3}$

${ }^{1}$ Programa de Pós-graduação em Tecnologias Limpas, Centro Universitário de Maringá - UniCesumar, Avenida Guedner, 1610, 87050-900, Maringá-PR.

(jose.goncalves@unicesumar.edu.br)

${ }^{2}$ Centro Universitário de Maringá - UniCesumar, Avenida Guedner, 1610, 87050900, Maringá-PR.

${ }^{3}$ Instituo Cesumar de Ciência, Tecnologia e Inovação - ICETI, Avenida Guedner, 1610, 87050-900, Maringá-PR.

\section{Recebido em: 08/04/2017 - Aprovado em: 10/06/2017 - Publicado em: 20/06/2017} DOI: 10.18677/EnciBio_2017A152

\begin{abstract}
RESUMO
As questões sobre sustentabilidade têm ganhado cada vez mais espaço nas legislações e selo de certificação nos diferentes países, grande parte devido aos impactos ambientais, oriundos principalmente, da indústria da construção civil. Assim, o objetivo foi avaliar o grau de conhecimento das principais construtoras de Maringá-PR sobre a certificação ambiental norte-americana LEED (Leadership in Energy and Environmetal Design), bem como identificar as principais dificuldades para sua implementação nos empreendimentos. Para tal, foram selecionadas 11 construtoras premiadas pelo SINDUSCON (Serviço Social da Indústria da Construção Civil) entre 2010 e 2016, destas apenas quatro concordaram em participar da pesquisa. A pesquisa foi realizada mediante aplicação de questionário onde se obteve informações gerais sobre a construtora e respostas específicas sobre certificação LEED e sustentabilidade no setor da construção civil. Verificou-se que todas construtoras conhecem a importância da sustentabilidade da construção civil. Porém, embora as mesmas já possuam empreendimentos baseados em ações da sustentabilidade, estas compreendem basicamente mecanismos relacionados com eficiência hídrica, energética e gestão de resíduos. Das quatro construtoras entrevistadas, apenas as B e D conheciam a Certificação LEED. Entretanto apenas a construtora D possuía empreendimentos que já foram Certificados LEED. Todas as construtoras se consideraram sustentáveis, $e$ as construtoras $B$ e $D$ demonstraram maior conhecimento ao tema, assim como maior quantidade de ações em prol da sustentabilidade. A construtora A demonstrou pouco conhecimento e interesse pelo tema, já a construtora $C$ justificou seu baixo número de ações sustentáveis pelo fato de concretizar obras de terceiros, executando exatamente 0 projeto pedido pelo cliente.
\end{abstract}

PALAVRAS-CHAVE: Sustentabilidade. LEED. Construção civil. 


\title{
CIVIL CONSTRUCTION AND ENVIRONMENTAL CERTIFICATION: STUDY OF THE IMPORTANCE OF SUSTAINABILITY IN CIVIL CONSTRUCTION BASED ON LEED CERTIFICATION
}

\begin{abstract}
Sustainability issues have been gaining more space in the legislations and certification seal in the different countries, largely due to the environmental impact, mainly from the construction industry. Thus, the objective was to evaluate the degree of knowledge of the principal builders of Maringá-PR on the North American environmental certification LEED (Leadership in Energy and Environmental Design), as well as to identify the main difficulties for its implementation in the ventures. For this, 11 contractors were selected by SINDUSCON (Social Service of the Construction Industry) between 2010 and 2016, of which only four agreed to participate in the research. The research was carried out through the application of a questionnaire that provided general information about the Construction Company and specific answers about LEED certification and sustainability in the civil construction sector. It was verified that all construction companies are aware of the importance of the sustainability of construction. However, although they already have ventures based on sustainability actions, they basically comprise mechanisms related to water efficiency, energy and waste management. Of the four contractors interviewed, only $B$ and $D$ knew the LEED Certification. However, only the construction company D owned projects that were already LEED certified. All the constructors considered themselves sustainable, and the constructors B and D demonstrated a greater knowledge of the subject, as well as a greater number of actions for sustainability. Construction company a demonstrated little knowledge and interest in the subject, since construction company $\mathrm{C}$ justified its low number of sustainable actions due to the fact that it executed works by third parties, executing exactly the project requested by the client.
\end{abstract}

KEYWORDS: Sustainability. LEED. Construction.

\section{INTRODUÇÃO}

A temática do desenvolvimento sustentável começou a surgir na segunda metade do século $X X$, quando o homem começou a ter consciência da progressiva degradação infligida por suas políticas de desenvolvimento ao meio ambiente, e é cada vez mais um tema em discussão. Nesse período, que correspondeu ao final dos anos 1960 e início dos anos 1970, o progresso tecnológico ficou um pouco desacreditado, pois passou a ser considerada essencial para o bem-estar humano a convivência em harmonia com a natureza. Constatou-se que a biodiversidade na Terra está diminuindo em cerca de 50.000 espécies por ano (YEANGKEN, 2001).

Com a conscientização de que o desenvolvimento ancorado apenas em políticas econômicas sobrecarregará com o tempo o poder de autorregulação do sistema e a elasticidade, ou capacidade de assimilação, de outras espécies e dos sistemas naturais do planeta, conduzindo inevitavelmente à devastação total do meio natural, como se conhece hoje. Para proporcionar um desenvolvimento econômico e regional sem agredir o meio ambiente, medidas de desenvolvimento sustentável, devem ser realizadas (RIBEIRO \& MÜYLDER, 2014; LAZARO \& GREMAUD, 2017).

O desenvolvimento sustentável tem como meta incrementar a qualidade de vida (e não só o crescimento econômico), a equidade entre as pessoas no presente, incluindo a prevenção da pobreza, a equanimidade entre gerações. Gerações 
futuras merecem um ambiente tão bom quanto aquele de que se usufrui atualmente, senão melhor, com as preocupações relacionadas às problemáticas sociais, sanitárias e éticas do bem-estar humano (ARAUJO \& PEDROSA, 2014; SIQUEIRA et al., 2017).

Nesse contexto, a construção civil, apresenta-se hoje como um dos maiores consumidores dos recursos naturais no ambiente (FRAGA et al., 2016), consumindo $16,6 \%$ do fornecimento mundial de água pura, $25 \%$ da retirada de madeira e $40 \%$ de seus combustíveis fósseis e matérias manufaturadas (WINES, 2006). Desta forma, ela deve se readequar e aplicar técnicas sustentáveis a fim de lapidar sua metodologia, proporcionando uma integração entre 0 ambiente natural e 0 construído, acrescentando qualidade de vida aos seus usuários sem degradar desnecessariamente o ambiente.

A fim de aprimorar essa metodologia, foi desenvolvida a certificação LEED. LEED é o acrônimo de "Leardership in Energy and Environmental Design" (Liderança em Energia e Design Ambiental), um sistema voluntário de certificação para edifícios sustentáveis, desenvolvido pela organização norte-americana "United States Green Buiding Council" (USGBC). Sua primeira versão foi disponibilizada em 1998 nos Estados Unidos, derivada de um movimento de engenheiros e arquitetos insatisfeitos com a demanda oriunda do mercado e clientes. Insatisfação essa proveniente de um conhecimento e desejo de contribuir para o planeta com projetos mais sustentáveis (USGBC, 2009; USGBC, 2015).

O sistema LEED fornece uma estrutura clara e concisa para identificação e implementação de medidas práticas e mensuráveis no projeto, construção, operação e manutenção de "Green Buildings". Utilizando-se de flexibilidade, possibilita, às equipes de projeto, decidir a melhor maneira de atender às suas exigências. $O$ selo certifica edifícios a partir de uma lista de pré-requisitos e créditos, e possui quatro níveis: Certified, Silver; Gold, Platinum em função do número de pontos alcançados (USGBC, 2009; USGBC, 2015).

Hoje, o Brasil ocupa a terceira posição no ranking mundial de países com mais processos de Certificação LEED, com 957 projetos registrados e 224 certificados, atrás dos Estados Unidos, detentores da primeira posição com 52.921 projetos registrados e 21.965 certificados, seguidos pela China que ocupa a segunda posição, com 1.936 projetos registrados e 583 certificados (USGBC, 2009; USGBC, 2015). Em nível regional o município de Maringá-PR conta com apenas um empreendimento com o selo, a fábrica da empresa Coca-Cola FEMSA BRASIL, nesse caso o nível da certificação alcançada foi a "silver", pois a empresa atingiu a pontuação entre 50 e 59 pontos.

Diante de tais informações, questionam-se os reais benefícios da obtenção da certificação LEED em empreendimentos brasileiros, sejam estes ambientais, sociais e econômicos, para as empresas que buscam a certificação (COSTA \& MORAES, 2013). Neste sentido, o objetivo do trabalho foi avaliar o grau de conhecimento das principais construtoras de Maringá-PR sobre a certificação ambiental norteamericana LEED (Leadership in Energy and Environmetal Design), bem como identificar as principais dificuldades para a implementação da certificação em seus empreendimentos.

\section{MATERIAL E METODOS}

O presente trabalho foi realizado mediante pesquisa bibliográfica no campo da sustentabilidade e certificações ambientais no setor industrial e na construção civil. Foram analisadas as estratégias que devem ser abordadas em um projeto, bem 
como execução de uma construção, para que esta possa ser denominada como "green building", através de uma visita técnica na empresa COCA COLA FEMSA BRASIL, localizada na cidade de Maringá-PR, pois a referida empresa possui Certificação LEED SILVER em seu estabelecimento, contribuindo desta forma para aprimoramento desta pesquisa.

A pesquisa foi realizada em construtoras na cidade de Maringá-PR. O critério adotado para a escolha das construtoras foi o prêmio Serviço Social da Indústria da Construção Civil (SINDUSCON), sendo analisados dados desde o primeiro prêmio, em 2010, até o ultimo, na data de dezembro de 2016. Todas as empresas foram contatadas, entretanto o questionário foi aplicado em quatro das 11 construtoras ganhadoras de um ou mais prêmios.

A coleta de dados foi realizada mediante a aplicação de um questionário respondido pelos engenheiros das respectivas construtoras, contendo 20 questões fundamentadas nos principais segmentos do "checklist" da Certificação LEED, como: terrenos sustentáveis, uso racional da água, energia e atmosfera; qualidade do ambiente interno e inovação de projeto.

A análise das respostas obtidas a partir dos questionários foi embasada nos conhecimentos adquiridos pelo pesquisador Felipe Ferreira Cossich no curso "COMO SE TORNAR UM LEED GA", reconhecido pelo USGBC (United States Green Building Council), que capacita seus participantes como profissional LEED GA, garantindo capacitação profissional perante a Certificação LEED e credenciando-o para trabalhar e prestar consultorias em construções que buscam essa certificação, bem como através das observações realizadas na empresa COCA COLA FEMSA BRASIL que possui Certificação LEED SILVER.

\section{RESULTADOS E DISCUSSÃO}

A pesquisa foi realizada na cidade de Maringá, Norte do Estado do Paraná, na latitude: -23.4273, longitude: $-51.9375,23^{\circ} 25^{\prime} 38^{\prime \prime}$ Sul, $51^{\circ} 56^{\prime} 15^{\prime \prime}$ Oeste, com área de $487,052 \mathrm{~km}^{2}$ e população estimada de 403.063 habitantes segundo o IBGE em 2016. Maringá é hoje o terceiro maior município do estado do Paraná e o sétimo da região Sul do país. Conhecido como cidade verde pela sua área urbana densamente arborizada, o município está entre os melhores do país em qualidade de vida, fomentando, assim, a discussão sobre o tema sustentabilidade.

Os dados da pesquisa foram obtidos por meio de questionários submetidos às construtoras, vencedoras do prêmio SINDUSCON-NOR, abordando as seguintes categorias: Obras de Terceiros, Obras Públicas, Incorporações. Sendo o SINDUSCON-NOR, o sindicato que representa as empresas do setor da construção civil em 109 municípios da região Noroeste do Estado do Paraná. No entanto, dentre as construtoras contatadas, apenas quatro construtoras se dispuseram a participar deste trabalho, sendo denominadas "Construtora A", "Construtora B", "Construtora C" e "Construtora D" (Quadro 1).

QUADRO 1- Principais características das construtoras pesquisadas.

\begin{tabular}{lccc}
\hline Identificação & $\begin{array}{c}\text { Ano de } \\
\text { fundação }\end{array}$ & $\begin{array}{c}\text { Local de } \\
\text { origem }\end{array}$ & No de funcionários \\
\hline Construtora A & 1992 & Maringá-PR & 433 \\
Construtora B & 1975 & Maringá-PR & 150 \\
Construtora C & 1999 & Maringá-PR & 45 \\
Construtora D & 1970 & Londrina-PR & 2.000 \\
\hline
\end{tabular}


Todas as construtoras participantes da pesquisa têm atividades consolidadas no setor da construção civil, sendo que as construtoras A e B com obras sendo executadas em Maringá e em algumas outras cidades do estado do Paraná, a construtora $\mathrm{C}$ somente em Maringá e a construtora D apresenta obras em todo território brasileiro.

O setor construtivo tem grande influência sobre os impactos ambientais, por esse motivo, a sustentabilidade na construção civil é tão almejada, sempre apoiada em seus três pilares: ambiental, econômico e social. A respeito dessa temática, $100 \%$ das construtoras entrevistadas têm consciência de que a sustentabilidade é o futuro da construção civil e logo será uma tendência do mercado. Entretanto, apenas a construtora $A$ e $D$ citaram ações das empresas em prol da sustentabilidade como: utilização de uma cartilha com os processos sustentáveis utilizados e desenvolvidos em obras; monitoramento de indicadores referentes ao consumo de água, de energia e resíduos; utilização de máquina para triturar resto de concreto e alvenaria em obra, minimizar os impactos ambientais e consumir cada vez menos recursos.

De acordo com CAVALCANTE (2011) o setor construtivo é responsável por grande parte dos impactos ambientais causados pelo homem, seja em nível mundial ou nacional. Quando abordado o quesito poluição na etapa construtiva, todas as construtoras participantes demonstraram preocupações básicas com a destinação correta dos resíduos sólidos da construção civil.

A construtora B tem consciência de que o sistema construtivo convencional gera muitos resíduos, então opta por sistemas menos impactantes como o "dry-wall". A construtora $\mathrm{D}$, além dessa consciência, classificou todas as suas ações referentes à poluição na etapa construtiva, relatando que todo entorno da obra recebe um estudo e cuidados especiais como benfeitorias para redução de impactos, canteiros reaproveitáveis, segregação de resíduos e reaproveitamento de resíduos, contribuindo para um canteiro mais saudável.

No que tange, a escolha do terreno para a construção, a Certificação LEED prioriza alguns fatores que qualificam um terreno como ideal para construção de um "green building". Critérios como o clima local do projeto; desenvolvimento prévio do terreno; se o mesmo está conectado à infraestrutura e transporte público local; quais espécies (animal e vegetal) presentes utilizam a área como habitat e como elas poderão se adaptar com as mudanças realizadas no terreno; como o projeto pode contribuir com a comunidade; onde as pessoas da área vivem e trabalham e como se locomovem (USGB, 2009; USGB, 2015). Ainda não totalmente engajadas no conceito LEED, as construtoras avaliadas mostraram-se apoiadas no pilar econômico da sustentabilidade, evidenciando que apenas o fator financeiro e o de mercado são levados em conta no momento da escolha do terreno, deixando, dessa forma, os pilares ambientais e sociais em segundo plano.

Entre as respostas obtidas, a construtora $D$ foi a que mais apoiou nos conceitos da sustentabilidade, mostrando consciência em relação à economia de água e percepção da necessidade de eficiência no consumo desta. Para atender a esse objetivo, a empresa relatou que está em processo de desenvolvimento para quantificação de consumo de água na execução de suas obras e também na obra finalizada. Essas observações encontram-se em conformidade com os conceitos abordados pela Certificação LEED, quando tratados os pré-requisitos e créditos de eficiência hídrica.

Todas as construtoras demonstraram dispor de recursos para economia de água em seus empreendimentos, destacando, entre eles, a captação de água pluvial, registros com temporizador, medidores de água individuais, vasos com caixa 
acoplada, conscientização dos funcionários, monitoramento de consumo e aeradores em torneiras. Mesmo ficando evidente a importância da economia de água na construção civil, nenhuma das construtoras entrevistadas dispõe de dados que contabilizem a economia que suas ações promovem.

No que se refere, a energia elétrica, apesar de não fazerem uso de fontes renováveis de energia, as empresas utilizam mecanismos para diminuir o consumo. Os principais mecanismos apresentados estavam relacionados à iluminação natural, ventilação natural e cruzada, preocupação com a orientação do edifício quanto ao sol, utilização de temporizador de luz e sensores de presença, lâmpadas led e equipamentos mais eficientes com o menor gasto energético. Do mesmo modo que não dispõem dos dados referentes à economia de água, as construtoras também desconhecem 0 real potencial de economia de energia elétrica de seus empreendimentos.

Os aparelhos de resfriamento utilizados pelas construtoras costumam apresentar os gases CFC e HCFC em seus sistemas de resfriamento de ar, como sabemos estes gases são altamente poluidores e danosos à camada de ozônio (BRASIL, 2014). Quando questionados sobre quais gases são utilizados em seus aparelhos de resfriamento, nenhuma das construtoras soube informar. Este dado mostra a falta de organização profissional sobre o assunto.

Outra temática abordada foi o gerenciamento de resíduos. O setor da construção civil é responsável, por gerar um volume significativo de resíduos de construção e demolição (PASCHOALIN-FILHO et al., 2016) que, quando descartados, inadequadamente, depositados em encostas de rios, vias e logradouros públicos, terrenos baldios podem ocasionar diversos impactos ao meio ambiente (ALVES , 2015; PASCHOALIN-FILHO et al., 2016), resultando em prejuízos aos cofres municipais e à saúde pública (SILVA et al., 2013).

De acordo com a lei federal 12.305 de 2010, (BRASIL, 2010) todas as empresas geradoras de resíduos são obrigadas a possuir um plano de gerenciamento de resíduos sólidos (PGRS). Todas as construtoras analisadas neste estudo dispõem do PGRS, cada plano tem suas características particulares, entretanto as construtoras listaram algumas ações integrantes de seus planos, referentes ao gerenciamento de resíduos sólidos. Dentre estes destacam-se: o controle de entrada e saída de resíduos, descarte adequado com empresas credenciadas pela prefeitura, a separação e triagem dos resíduos, e a reciclagem dos resíduos na própria obra.

Seguindo as premissas da Certificação LEED, os resíduos sólidos da construção civil devem ser desviados dos aterros sanitários, uma vez que estes possuem vida útil, que pode ser diminuída com a presença de resíduos da construção civil. No entanto, nenhuma das construtoras pesquisadas possuía dados quantitativos referentes ao volume de resíduos desviados dos aterros sanitários.

Quanto aos indicadores de qualidade do ar para funcionários e futuros usuários, apenas as construtoras B e D mostraram ações no ambiente de trabalho. Porém, apenas a construtora $D$ expôs ações concretas (ventilação natural e mecânica) para os usuários, mostrando-se muito abaixo dos conceitos de qualidade do ar, descritos por COLBECK et al. (2010) e AGRAWAL (2012).

A certificação LEED contempla ainda questões referentes à inovação de projetos. Para RIGHETTI \& PALLONE (2007), o termo inovação tecnológica é definido pela introdução no mercado de um produto ou de um processo produtivo tecnologicamente novo ou substancialmente aprimorado. 
As empresas que mais se destacaram em inovações foram as construtoras $B$ e D. A primeira buscando novos métodos construtivos, e a segunda mostrando-se pioneira em tecnologias como o escoramento metálico, layout de canteiro, empreendimentos eficientes e sendo ainda a única construtora que já executou obra que recebeu certificação LEED (fábrica da Coca Cola FEMSA Brasil, localizada na cidade de Maringá-PR).

Em relação à valorização e desenvolvimento social e econômico regional veiculados com a aquisição de matérias e/ou produtos da própria região, entre as entrevistadas, apenas as construtoras B, C e D mostraram-se preocupadas em valorizar produtos regionais, com intuito de fomentar a economia local, além da redução de impactos ambientais referentes ao deslocamento de produtos e minimização de frete. No que tange à percepção dos clientes, relacionado ao diferencial proporcionado por um "green building", as empresas relataram que a procura por esse tipo de edificação ainda é pequena, salvo casos de clientes específicos como foi o caso da fábrica da Coca Cola para a construtora D.

No final da pesquisa foram realizados questionamentos sobre as principais dificuldades em formalizar um projeto sustentável no setor da construção civil. Nesta pesquisa três construtoras relataram a barreira financeira (custo inicial) como maior dificuldade em formalizar um projeto sustentável. Esses relatos corroboram com KIBERT (2002), em que o autor reporta que o primeiro obstáculo encontrado é o maior custo de capital (custo inicial) de um projeto sustentável. Além disso, a construtora $\mathrm{D}$ ressaltou ainda que barreiras técnicas também dificultam o processo, fato este que está de acordo com o observado por MATHEUS (2009).

As construtoras $\mathrm{A}, \mathrm{B}$ e $\mathrm{C}$ afirmaram estar dispostas a fazer um investimento inicial de até $15 \%$, acrescidos do valor do empreendimento, para construir edificações sustentáveis e certificadas. Já a construtora $D$ avalia como viável economicamente qualquer valor adicional, desde que haja demanda de mercado.

Embora todas as empresas já possuam empreendimentos baseados nas questões da sustentabilidade, pode-se perceber que essas questões compreendem três itens básicos: mecanismos de eficiência hídrica, energética e gestão de resíduos. Das quatro construtoras entrevistadas, apenas as construtoras $B$ e $D$ conheciam a Certificação LEED. Entretanto apenas a construtora D possuía empreendimento que já recebeu a Certificação LEED.

Realizados os questionamentos, percebeu-se que todas as construtoras se consideram sustentáveis, o que não pode ser considerado uma afirmação verdadeira. As construtoras $B$ e $D$ demonstraram maior conhecimento ao tema, assim como maior quantidade de ações em prol da sustentabilidade. A construtora $A$ demonstrou pouco conhecimento e interesse pelo tema, já a construtora $C$ justificou seu baixo número de ações sustentáveis pelo fato de concretizar obras de terceiros, executando exatamente o projeto pedido pelo cliente.

Apesar de o Brasil ocupar o quarto lugar entre os países que mais possuem construções sustentáveis e certificadas LEED, fica claro que os conceitos de "green buildings" são preponderantes nas grandes capitais do país. Assim, este conceito de construções sustentáveis e certificadas, muitas vezes chega por meio de empresas estrangeiras e são impostos aos construtores e empreendedores brasileiros.

Aos poucos a construção sustentável vai criando raízes brasileiras e se adaptando à realidade do país; se por um lado a difícil mensuração dos benefícios ainda causa ceticismo, por outro, não há como refutar o reconhecimento e ganhos em mídia com fortalecimento da imagem da marca e até mesmo em competitividade atrelados à execução dessas edificações. A percepção brasileira de que essas 
edificações têm um custo inicial maior é real, mas ainda está embasada em pouco conhecimento, apenas em sentimento, o que confirma que as barreiras técnicas e sociais são mais impactantes do que as barreiras financeiras (COSTA \& MORAES, 2013).

\section{CONCLUSÕES}

Ao longo deste trabalho, pôde-se perceber o quão abrangente é o tema sustentabilidade na construção civil. Entretanto nota-se ainda que o pilar econômico da sustentabilidade infelizmente é o de maior relevância para os construtores, empresas, empreendedores e clientes. Outro fator preponderante para o sucesso desses empreendimentos em todo o território nacional e não só nas capitais é a divulgação dos benefícios para os usuários finais, fazendo uso de marketing verde, embasado em dados técnicos e os mais relevantes em questões quantitativas. Realizado o estudo na cidade de Maringá-PR, pôde-se concluir que a empresa Coca Cola FEMSA BRASIL conhece e consegue mensurar os benefícios das construções sustentáveis e da Certificação LEED, mostrando-se preocupada com os três pilares da sustentabilidade.

As construtoras de maior destaque e prestígio da cidade de Maringá-PR demonstraram possuir conhecimento sobre os benefícios e importância da sustentabilidade na construção civil, destacando ações básicas realizadas em seus empreendimentos, e algumas vezes demonstraram não conhecer a real importância dessas ações, como no caso da qualidade do ar em obras e empreendimentos. De maneira geral, de todas as construtoras entrevistadas, a construtora $D$ (que já possuía a Certificação LEED em sua obra) mostrou-se avançada em relação às demais. Entretanto, assim como as outras construtoras, ela precisa superar barreiras técnicas e sociais, destacando-se, por exemplo, quantificar o real potencial de economia de seus empreendimentos, quando tratados os temas consumo de água e energia.

\section{REFERÊNCIAS}

AGRAWAL, S. Effect of indoor air pollution from biomass and solid fuel combustion on prevalence of self-reported asthma among adult men and women in India: findings from a nationwide large-scale cross-sectional survey. Journal Asthma, v. 49, n.4, p. 355-65, 2012. Disponível em: <https://www.ncbi.nlm.nih.gov/pubmed/22397465>. Doi: 10.3109/02770903.2012.663030

ALVES, J. Resíduos da construção civil em obras novas. Interfaces CientíficasExatas e Tecnológicas, v. 1, n. 1, p. 53-65, 2015. Disponível em:<https://periodicos.set.edu.br/index.php/exatas/article/view/1812/1078>.

ARAUJO, M. F. F.; PEDROSA, M. A. Desenvolvimento Sustentável e Concepções de Professores de Biologia em Formação Inicial. Revista Ensaio Pesquisa em Educação em Ciências, v. 16, n. 2, p. 71-83, 2014. Disponível em: <http://www.scielo.br/pdf/epec/v16n2/1983-2117-epec-16-02-00071.pdf>. Doi: 10.1590/1983-21172014160204 
BRASIL. Ministério do Meio Ambiente, Departamento, de Mudanças Climáticas. Coordenação de Proteção da Camada de Ozônio. Ações brasileiras para a proteção da camada de ozônio CDU (2.ed.) 551.510.534. Brasília, DF: MMA, 2014. Disponível em: <http://simat.mma.gov.br/acomweb/Media/Documentos/6cafe5f80b94-4bd7-b.pdf>

BRASIL. Ministério do Meio Ambiente. Institui a política nacional de resíduos sólidos; altera a lei n. 9.605 de 12 fevereiro de 1998; e dá outras providências. Brasília, DF: $\quad 2010 . \quad$ Disponível em: <http://www.mma.gov.br/port/conama/legiabre.cfm?codlegi=636>

CAVALCANTE, I. G. Materiais Construtivos, Sustentabilidade e Complexidade Análise da Relação entre Especificações de materiais Construtivos e Desenvolvimento Sustentável. Dissertação de Mestrado - Programa de PósGraduação em Ciência Ambiental - USP, Universidade de São Paulo, São Paulo, 248 p, 2011.

COLBECK, I.; NASIR, Z. A.; ALI, Z. The state of indoor air quality in Pakistan--a review. Environmental Science and Pollution Research, v. 17, n. 6, p. 1187-96, 2010. Disponível em: < https://www.ncbi.nlm.nih.gov/pubmed/20162371>. Doi: 10.1007/s11356-010-0293-3.

COSTA, D.; MORAES, C.S.B. Construção civil e a certificação ambiental: análise comparativa das certificações LEED (Leadership in energy and environmental design) e AQUA (alta qualidade ambiental). Engenharia ambiental: Pesquisa e tecnologia, $\quad$ v. $10 . \quad$ n. $\quad 3, \quad$ p.160-169, $2013 . \quad$ Disponível em:<http://ferramentas.unipinhal.edu.br/engenhariaambiental/viewarticle.php?id=102 $0>$.

FRAGA, Y. S. B.; BARBOSA, A. Q.; SANTOS, L. H. P.; MOTA, W. V.; DORTAS, I. S. Tecnologia dos materiais: a utilização do tijolo de solo-cimento na construção civil. Caderno de Graduação-Ciências Exatas e Tecnológicas, v. 3, n. 3, p. 11, 2016.

Disponível em: <https://periodicos.set.edu.br/index.php/cadernoexatas/article/view/2882/1944>

KIBERT, C. J. Policy instruments for a Sustainable Building Built Environment. Journal of land use and Environmental Law, v. 17, n. 2, p. 379-394, 2002. Disponível em: <https://litigationessentials.lexisnexis.com/webcd/app?action=DocumentDisplay\&crawlid=1\&srctype= smi\&srcid $=3 B 15 \&$ doctype $=$ cite\&docid $=17+\mathrm{J} .+$ Land + Use $+\% 26+$ Envtl. + Law $+379 \& k e y$ $=50 \mathrm{~b} 96 \mathrm{f} 87 \mathrm{c} 9 \mathrm{f} 2 \mathrm{fc} 3 \mathrm{ac} 9 \mathrm{e} 3 \mathrm{f} 93 \mathrm{~d} 92251308>$

LAZARO, L. L. B; GREMAUD, A. P. Contribuição para o desenvolvimento sustentável dos projetos de Mecanismo de Desenvolvimento Limpo na América Latina. Revista Organizações \& Sociedade, v. 24, n. 80, 53-72, 2017. Disponível

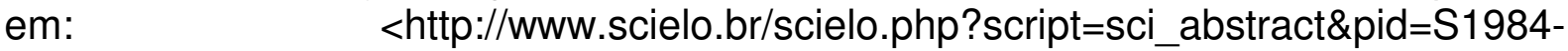
92302017000100053\&lng=en\&nrm=iso\&tlng=pt>. Doi: 10.1590/1984-9230803 
MATHEUS, R. Avaliação da sustentabilidade na construção civil: propostas para o desenvolvimento de edifícios mais sustentáveis. 2009. 427f. Tese (Doutorado em Engenharia Civil) - Universidade do Minho, Portugal, 2009.

PASCHOALIN-FILHO, J. A.; FARIA, A. C.; PIRES, G. W. M. O. \& LIMA-DUARTE, E. B. Investimentos em ativos imobilizados para instalação de usina de reciclagem de resíduos de construção civil de médio porte na zona leste de São Paulo. Desenvolvimento em Questão, v. 14, n.36, p. 320-351, 2016. Disponível em:

$<$ https://www.revistas.unijui.edu.br/index.php/desenvolvimentoemquestao/article/view /4428>. Doi: 10.21527/2237-6453.2016.36.320-351

RIBEIRO, S. D.; MÜYLDER, C. F. Economia Solidária - Em busca dos elementos essenciais da sustentabilidade e solidariedade. Revista Organizações \& Sociedade. v. 21, n. 71, p. 581-614, 2014. Disponível em: $<$ http://www.scielo.br/scielo.php?pid=S1984-

92302014000400581\&script=sci_abstract>. Doi: 10.1590/S198492302014217100004

RIGHETTI, S.; PALLONE, S. Consolidando também o conceito de inovação tecnológica. Inovação Uniemp, v. 3, n. 4, p. 26-27, 2007. Disponível em: $<$ http://inovacao.scielo.br/scielo.php?script=sci_arttext\&pid=S1808-

23942007000400014\&lng=es\&nrm=iso>

SILVA, L. M.; BARROS-JUNIOR, R. O.; MARQUES, L. C. A.; LIMA, A. M. M.; PONTES, A. N. Gestão de resíduos na construção civil: análise da aplicação dos recursos financeiros na região metropolitana de Belém- Pará. Enciclopédia Biosfera, v.9, n.17; p. 3506-3516, 2013. Disponível em:< http://www.conhecer.org.br/enciclop/2013b/MULTIDISCIPLINAR/gestao\%20de\%20r esiduos\%20na.pdf>

SIQUEIRA, S. M. C.; JESUS, V. S.; SANTOS, E. N. B.; WITHAKER, M. C. O.; SOUSA, B. V. N.; CMARGO, C. L. Atividades extensionistas, promoção da saúde e desenvolvimento sustentável: experiência de um grupo de pesquisa em enfermagem. Escola Anna Nery, v. 21, n. 1, p. 1-7, 2017. $<$ http://www.scielo.br/scielo.php?pid=S1414-

81452017000100701\&script=sci_abstract\&tlng=pt>. Doi: 10.5935/14148145.20170021

USGBC. Green Building Council. US: Certified Project Directory, 2015. Disponível em <http://www.usgbc.org/LEED/Project/CertifiedProjectList.aspx>.

USGBC. Green Building Council. Guia de Estudo de LEED AP Projeto e Construção de Edifício do USGBC (USGBC LEED AP Building Design + Construction Study Guide), Washington, 2009.

WINES, J. Green Architecture. Milan: Taschen, Brookings Institution, 2006.

YEANGKEN, K. El rasicacielos ecológico. Barcelona: Editorial GustavaGilli, 2001. 